\title{
Endoscopic pharyngeal pouch stapling: A retrospective study of 55 patients comparing intubation difficulty and body mass index as factors for success.
}

\section{Introduction}

Open and endoscopic approaches to pharyngeal pouch surgery have developed over more than a century and endoscopic methods now include electrocoagulation, laser division, and stapling. This approach is associated with a shorter post-operative hospital stay and a faster return to oral dietary intake ${ }^{1}$ whereas open surgery has a higher complication rate but may yield lower rates of recurrence ${ }^{2}$. Many patients have multiple comorbidities and so a lowerrisk endoscopic procedure with shorter anaesthetic time is preferable to reduce postoperative morbidity. However, as endoscopic surgery has a technical failure rate of $9.7 \%$ $18.9 \%^{3,4}$, patient selection is key in preventing repeated anaesthesia and maximising theatre utility.

There is currently no tool to aid selection of patients appropriate for a primary open pouch repair. One possible criterion is pouch size as some studies suggest that a small pouch could contribute to a failed endoscopic procedure ${ }^{5}$. Another is patient age, as recurrence rates may be higher if treated endoscopically as opposed to open surgery (13\% versus $6.5 \%)^{4}$, making the latter a potentially better option in younger patients.

In this paper, we consider whether the following factors might affect the success of endoscopic pouch stapling:

1. Size of pharyngeal pouch

2. Mouth opening

3. Intubation difficulty

4. Body mass index (BMI) 


\section{Methods}

\section{Ethical considerations}

No formal ethical approval was required as data collection was part of an audit and distribution remained anonymous throughout.

\section{Study design}

Data was collected retrospectively for pharyngeal pouch surgery performed by a single surgeon between January $1^{\text {st }} 2013$ and $31^{\text {st }}$ December 2016 at a tertiary centre. The first attempted visualisation of the pouch was using the Oxford Universal Pouch scope (Roberts Surgical Healthcare Ltd. Kidderminster, United Kingdom) which is designed so that a Hopkins fibre-optic rod can be inserted alongside the stapling gun. If unable to pass this scope then the Weerda distending pharyngoscope was second choice. Third-choice was a Negus pharyngoscope and a failed procedure was defined as the inability to pass either of these scopes or inability to engage the staple anvil.

Records were examined for anaesthetic documentation, patient demographics, surgical technique, outcome and details of any previous surgery. As well as procedure abandonment, failed endoscopic procedure was also defined as a recurrence of symptoms within 6 months alongside evidence of a persistent pouch. Patients were followed up until $31^{\text {st }}$ December 2018 to determine any re-presentations to clinic or if revision surgery was required. The preoperative contrast study was examined for each patient and pouch size was determined by the length of the anterior wall as this was the distance the staple engaged. Pouches were categorised according to the Morton System of small (less than 2cm), medium (between 2$4 \mathrm{~cm})$ and large (more than $4 \mathrm{~cm})^{6}$.

All endotracheal intubations were performed or supervised by a consultant anaesthetist. Anaesthetic records were examined for the Modified Mallampati score ${ }^{7}$ to grade mouth 
opening and the predicted ease of intubation prior to anaesthetic as well as the Modified Cormack-Lehane (MCL) score to indicate the grade of intubation difficulty ${ }^{8}$. A Chi-squared test was used to evaluate the association between endoscopic procedure failure and pouch size, mouth opening and intubation difficulty. We applied Bayes Rule to calculate the probability of staple failure based on prior evidence of failure rates alongside the new data from this study.

Body Mass Index $\left(\mathrm{BMI}, \mathrm{kg} / \mathrm{m}^{2}\right)$ was taken from pre-operative nursing assessments and categorised according to the World Health Organisation classification as underweight (less than 18.5), normal range (18.5-25), overweight (25-30), or obese (more than 30$)$.

\section{Results}

Fifty-five pharyngeal pouch procedures were performed during the four-year study period. Patients ranged from 40 to 91 years old, with an average age of 70 . The male to female ratio was 2:1.

The most common presenting complaints were of partial dysphagia and the regurgitation of food. Ten patients were classified as overweight or obese; these patients were more likely to present with cough and globus as their primary symptom.

\section{Primary open pouch repair}

This was done in two patients who were younger than the study average (aged 51 and 64). One patient was undergoing a scheduled thyroidectomy and so the open pouch procedure was done at the same time. The second patient opted for an open approach due to the lower rate of reported recurrence. The BMI of both patients was within normal range and there was no recurrence at the end of the study period.

Failure of endoscopic stapling 
Forty-seven patients underwent successful endoscopic stapling, of which, three patients were excluded from the study due to missing anaesthetic charts leaving us unable to determine the full investigative factors. Of the six patients who had a failed endoscopic approach, four were already consented for an open procedure on the same day and the remaining two were followed up in clinic but decided against an open repair. The endoscopic stapling failure rate for a small pouch was $6 \%$ and the failure rate for a medium and large pouch was $14 \%$ and $17 \%$ respectively (Table 1 ). There was no statistically significant difference ( $p=0.77$, Chi-Square test) in endoscopic failure rates between small, medium and large pouch sizes.

Modified Mallampati score was recorded in the endoscopic cases and although having a lower score was associated with a lower failure rate (Table 2), a range of scores were seen within the failed procedures (Table 3) and overall the result was not statistically significant ( $p=0.22$, Chi-Square with Yates' correction).

A MCL intubation grade of $2 b$ or above, as recorded in four cases, was universally associated with failed endoscopic stapling (Table 2); this result was statistically significant ( $p=0.0006$, Chi-Square with Yates' correction). Using Bayes Rule ( $A=$ Failed procedure, $\mathrm{B}=\mathrm{MCL}$ score of $>2 \mathrm{a}$ and $9.7 \%$ as the referenced failure rate) we calculated the probability of failed stapling to be $81 \%$ if Modified Cormack-Lehane score was $2 b$ or 3 .

\section{Recurrence of pouch}

The recurrence rate after pouch stapling was $6.8 \%(n=3)$, this was evidenced by recurrence of symptoms followed by a repeat barium swallow within the study period. In addition to this, 4 patients had already undergone pouch stapling at their local Trust within 3 years. Details for pouch recurrence patients are given in Table 4. The majority of patients had a high BMI.

\section{Discussion}

Synopsis of key findings 
Variations in patient anatomy can make insertion of a diverticuloscope difficult to the extent that some patients are unsuitable for an endoscopic procedure. Trismus, receding mandible and spinal disease will affect the intended straight axis between mouth, pharynx and oesophagus. As the larynx must be lifted anteriorly to expose the cricopharyngeus muscle, a posteriorly-positioned larynx/hypopharynx or an obstructed view from the epiglottitis may reduce the likelihood of successful pharyngeal pouch stapling and our series suggests that Modified Cormack-Lehane score is a sensitive predictor for failed endoscopic stapling. Therefore, reviewing previous anaesthetic charts for information on laryngeal views may help plan the approach. Whilst we did not assess neck extension and lower jaw protrusion, other studies showed these to be a useful predictor of difficult intubation and may be something to investigate in the future. ${ }^{9}$

The endoscopic stapler has a redundant distal lip of $5 \mathrm{~mm}$, and so in theory a pouch with a short height as measured on contrast swallow ought to be less amenable to stapling. However, in practice we did not find a significant failure rate in this group of patients or a significant recurrence rate. It is possible that the height measured on contrast swallow does not properly reflect pouch size. However, it is more likely that favourable conditions aligning the stapler help advance the staple anvil further.

The majority of patients presented with dysphagia and had a low or normal BMI, however the majority of revision stapling was performed on patients with a high BMI and whilst the reason for this is unclear, a combination of fibro-adipose deposition in the cricopharyngeus (seen on histological specimens from pharyngeal pouch patients ${ }^{6}$ ) and inflammation secondary to acid reflux, is thought to impair upper oesophageal sphincter function. This change in architecture along with increased intra-abdominal pressure could explain both the development of a pouch and its recurrence in this patient subpopulation. Given the rise in obesity and better access to radiological investigations, we predict that the demand for pharyngeal pouch surgery will increase. 


\section{Comparison with other studies}

There are many recommendations in the literature to perform open surgery for smaller pharyngeal pouches due to endoscopic failure rates ${ }^{6,10,11,12}$ however, anaesthetic and patient factors have never been co-investigated and pouch size did not affect stapling success in this study population. The overall staple failure rate was $11 \%$ which is similar to that already reported in the literature ${ }^{3,4}$.

\section{Study strengths}

To our knowledge this is the first study looking at patient weight, intubation difficulty and mouth opening as predictors for successful pharyngeal pouch stapling. We believe that awareness of these factors will help better plan operating time and optimise theatre efficiency.

\section{Study limitations}

This study only investigates data from a single surgeon. Whilst this limits the variable of operator skill, a larger data set is required to better predict stapling failure and to understand the association between high $\mathrm{BMI}$ and pouch recurrence.

\section{Keypoints}

- A Modified Cormack-Lehane score of $2 \mathrm{~b}$ or 3 has a predicted endoscopic stapling failure rate of $81 \%$ and so previous anaesthetic records may be useful in surgical planning.

- Small pouch size is not a predictor for failure when other factors are favourable, and endoscopic surgery should not be ruled out on this basis.

- Patients with a high BMI have a higher chance of pouch recurrence.

Conflicts of interest: None to declare. 


\section{Tables and Figures}

\begin{tabular}{|l|c|c|c|}
\hline $\begin{array}{l}\text { Pouch size } \\
(\mathrm{cm})\end{array}$ & $\begin{array}{c}\text { Number in } \\
\text { study }\end{array}$ & $\begin{array}{c}\text { Number of } \\
\text { failed stapling } \\
\text { procedures }\end{array}$ & $\begin{array}{c}\text { Number of } \\
\text { primary open } \\
\text { procedures }\end{array}$ \\
\hline Small $(<2)$ & 16 & 1 & 0 \\
\hline Medium (2-4) & 29 & 4 & 1 \\
\hline Large $(>4)$ & 7 & 1 & 1 \\
\hline Total & $\mathbf{5 2}$ & $\mathbf{6}$ & 2 \\
\hline
\end{tabular}

Table 1 - Failure of endoscopic stapling versus pouch size

\begin{tabular}{|c|c|c|c|c|c|}
\hline $\begin{array}{l}\text { MCL } \\
\text { score }\end{array}$ & $\begin{array}{c}\text { Number } \\
\text { of } \\
\text { patients }\end{array}$ & $\begin{array}{l}\text { Failure rate } \\
\text { for MCL } \\
\text { score }\end{array}$ & $\begin{array}{l}\text { Mall } \\
\text { Score }\end{array}$ & $\begin{array}{c}\text { Number } \\
\text { of } \\
\text { patients }\end{array}$ & $\begin{array}{l}\text { Failure rate } \\
\text { for Mall score }\end{array}$ \\
\hline 1 & 43 & $5 \%(n=2)$ & 1 & 31 & $3 \%(n=1)$ \\
\hline $2 a$ & 3 & $0 \%(n=0)$ & 2 & 17 & $24 \%(n=4)$ \\
\hline $2 b$ & 1 & $100 \%(n=1)$ & 3 & 2 & $50 \%(n=1)$ \\
\hline 3 & 3 & $100 \%(n=3)$ & & & \\
\hline
\end{tabular}

Table $2-$ MCL and Mall scores in study population. MCL = Modified Cormack-Lehane scale. Mall = Modified Mallampati score.

\begin{tabular}{|c|c|c|c|c|c|}
\hline Patient No. & $\begin{array}{c}\text { Pouch } \\
\text { size } \\
(\mathrm{cm})\end{array}$ & Mall & MCL & BMI & Failure details \\
\hline 12 & 1.6 & 2 & $2 \mathrm{~b}$ & 32 & Failure to engage anvil \\
\hline 18 & 2.0 & 2 & 3 & 27 & Failure to engage anvil \\
\hline 24 & 2.1 & 2 & 1 & 32 & Persistence of pouch \\
\hline 34 & 2.8 & 2 & 3 & 22 & Failure to engage anvil \\
\hline 43 & 3.9 & 3 & 3 & 23 & Failure to engage anvil \\
\hline 49 & 5.9 & 1 & 1 & 27 & Persistence of pouch \\
\hline
\end{tabular}

Table 3 - Details for patients who had failed endoscopic stapling. Mall = Modified Mallampati score, $\mathrm{MCL}=$ Modified Cormack-Lehane grade, $\mathrm{BMI}=$ Body Mass Index . 


\begin{tabular}{|c|c|c|c|c|c|}
\hline Patient No. & $\begin{array}{c}\text { Pouch } \\
\text { size }(\mathrm{cm})\end{array}$ & Mall & MCL & BMI & Details \\
\hline 1 & 1.0 & 1 & 1 & 32 & Patient's second procedure \\
\hline 8 & 1.4 & 2 & 1 & 32 & Recurrence within study period \\
\hline 25 & 2.3 & 2 & 1 & 18 & Patient's second procedure \\
\hline 28 & 2.5 & 2 & 1 & 32 & Patient's second procedure \\
\hline 30 & 2.5 & 1 & 1 & 40 & Recurrence within study period \\
\hline 44 & 4.0 & 1 & 1 & 34 & Patient's second procedure \\
\hline 50 & 6.0 & 2 & $2 \mathrm{a}$ & 27 & Recurrence within study period \\
\hline
\end{tabular}

Table 4 -Details for patients who had recurrence of pharyngeal pouch. Mall = Modified Mallampati score, $\mathrm{MCL}=$ Modified Cormack-Lehane grade, $\mathrm{BMI}=$ Body Mass Index. 


\section{References}

\footnotetext{
${ }^{1}$ Albers DV, Kondo A, Bernardo WM, et al. (2016) Endoscopic versus surgical approach in the treatment of Zenker's diverticulum: systematic review and meta-analysis.16;4:678-686.

${ }^{2}$ Agalato E, Jose J, England RJ. (2016) Is pharyngeal pouch stapling superior to open pharyngeal pouch repair? An analysis of a single institution's series. Laryngol Otol. 130:873-7.
}

${ }^{3}$ Harris RP, Weller MD, Porter MJ. (2010) A follow up audit of pharyngeal pouch surgery using endoscopic stapling. Eur Arch Otorhinolaryngol. 267:939-43.

${ }^{4}$ Verdonck J, Morton RP. (2015) Systematic review on treatment of Zenker's diverticulum. Eur Arch Otorhinolaryngol. 272:3095-107.

${ }^{5}$ Lieder A, Nasseri F, Sharma A. et al. (2008) Safety and efficacy of endoscopic pharyngeal pouch stapling in a large UK study. Clin Otolaryngol. 33:127-30.

${ }^{6}$ Siddiq MA, Sood S, Strachan D. (2001) Pharyngeal pouch (Zenker's diverticulum). Postgrad Med J. 77:506-11.

${ }^{7}$ Samsoon GL, Young JR. Difficult tracheal intubation: a retrospective study. Anaesthesia. 1987;42(5):487-90.

${ }^{8}$ Yentis, SM, Lee, DJ. (1998). "Evaluation of an improved scoring system for the grading of direct laryngoscopy". Anaesthesia. 53:1041-4.

9 UI Haq MI, Ullah H (2013) Comparison of Mallampati test with lower jaw protrusion maneuver in predicting difficult laryngoscopy and intubation. J Anaesthesiol Clin Pharmacol. 29(3): 313-317.

${ }^{10}$ Casso CD, Lalam M, Ghosh S, et al. (2006) Endoscopic stapling diverticulotomy: an audit of difficulties, outcome, and patient satisfaction. Otolaryngol Head Neck Surg. 2006 Feb;134(2):288-93.

11 Rizzetto C, Zaninotto G, Costantini M, et al. (2008) Zenker's diverticula: feasibility of a tailored approach based on diverticulum size. J Gastrointest Surg. 12(12):2057-64.

12 Gutschow CA, Hamoir M, Rombaux P, et al (2002). Management of pharyngoesophageal (Zenker's) diverticulum: which technique? Ann Thorac Surg. 74:1677-82. 